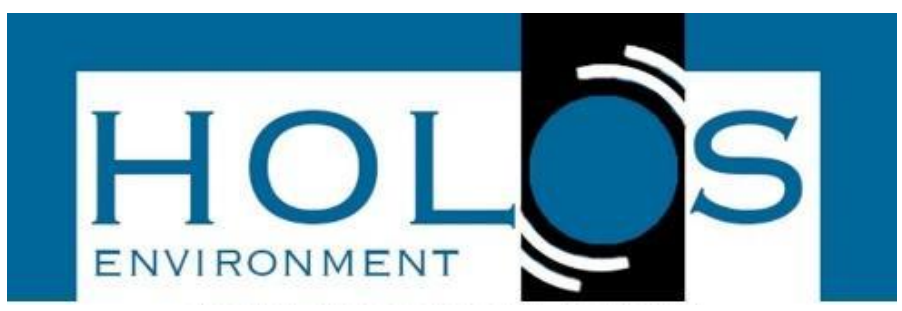

\title{
ESTUDOS DE ALTERNATIVAS LOCACIONAIS PARA ATERROS SANITÁRIOS NOS PROCESSOS DE LICENCIAMENTO AMBIENTAL NO ESTADO DE MINAS GERAIS
}

\section{LOCATIONAL ALTERNATIVES STUDIES OF LANDINGS IN ENVIRONMENTAL LICENSING PROCESSES OF MINAS GERAIS STATE}

\author{
Guilherme Henrique Santos Cruz¹; Maria Rita Raimundo e Almeida ${ }^{1}$
}

Artigo recebido em: 28/01/2020 e aceito para publicação em: 26/02/2020.

DOI: http://dx.doi.org/10.14295/holos.v20i2.12371

\begin{abstract}
Resumo: Aterro sanitário é uma tipologia de projeto causadora de significativo impacto, portanto, sujeita ao processo de Avaliação de Impacto Ambiental (AIA), em que o estudo de alternativas se desenvolverá para a escolha de uma opção de área adequada para construção do empreendimento. O presente trabalho objetivou avaliar como estão sendo contemplados os estudos de alternativas locacionais para construção de aterros sanitários nos processos de licenciamento com AIA no Estado de Minas Gerais (MG). A metodologia consistiu em investigar os processos de licenciamento ambiental de aterros em MG que consideraram o estudo de alternativas locacionais por meio de Estudo de Impacto Ambiental (EIA) disponíveis no Sistema Integrado de Informações Ambientais. Foram encontrados 1584 processos, sendo que 17 possuíam EIA, 8 estavam disponíveis e apenas 7 dispunham de estudo de alternativas locacionais, os quais foram analisados. Os estudos de caso, principalmente os critérios de seleção de áreas utilizados, foram comparados entre si e, posteriormente, comparados com a literatura. As comparações possibilitaram levantar quais os critérios que estão sendo utilizados e atestar que não existe uma metodologia modelo, resultando em diferentes formas de se adotar critérios e processos de seleção da área de implantação da atividade de disposição final dos resíduos.
\end{abstract}

Palavras-chave: Licenciamento ambiental. Avaliação de impacto ambiental. Estudo de alternativas locacionais. Aterros sanitários.

\begin{abstract}
Landfill is a typology of project causing significant impact, therefore, subject to Environmental Impact Assessment (EIA) process, in which the study of alternatives will be developed for choice of an appropriate area option for construction of the project. The present work aimed to evaluate how the studies of locational alternatives of landfills in licensing processes with EIA in the State of Minas Gerais (MG) - Brazil are being considered. The methodology consisted in investigating the environmental licensing processes of landfills in MG that considered the study of locational alternatives through the Environmental Impact Study (EIS) available in the Integrated Environmental Information System. A total of 1,584 cases were found, of which 17 had EIS, 8 were available and only 7 had a study of locational alternatives, which were analyzed. The case studies, especially the selection of areas criteria used, were compared to each other and later compared to literature. The comparisons made it possible to establish which criteria are being used and to attest that there is no model methodology, resulting in different ways of adopting criteria and processes for selecting the area for the final disposal of waste.
\end{abstract}

Keywords: Environmental licensing. Environmental impact assessment. Study of locational alternatives. Sanitary landfills.

\footnotetext{
${ }^{1}$ Universidade Federal de Itajubá (UNIFEI), Itajubá, MG. E-mail: (guihscruz@gmail.com,maria.rita.ralmeida@gmail.com)
} 


\section{INTRODUÇÃO}

A Avaliação de Impacto Ambiental (AIA) é um instrumento designado para auxiliar na busca de alternativas mais sustentáveis para o uso dos recursos naturais frente ao desenvolvimento da sociedade. Ela visa antever impactos ambientais ainda na fase de planejamento e concepção dos projetos, gerando resultados que auxiliam na tomada de decisão para concretização das opções que contemplem as melhores maneiras de se reduzir os impactos negativos e que se adequem de forma mais razoável ao ambiente local (IAIA, 1999).

Desde o seu surgimento, a AIA dispersou-se rapidamente como prática institucionalizada em âmbito mundial, devido ao seu potencial de utilização implicar em consequências benéficas ao desenvolvimento, no entanto, existem muitas críticas quanto à efetividade de seus procedimentos (MORGAN, 2012; ALMEIDA e MONTAÑO, 2017). Dentre as suas principais deficiências, encontra-se a consideração de alternativas para o projeto (POPE et al., 2013). Ainda, o trabalho de Duarte et al. (2017) apontou a deficiência dos estudos de alternativas ao avaliarem a percepção dos praticantes de AIA no Brasil quanto à importância da qualidade dos estudos de impacto ambiental. Estes, muitas vezes, têm sido desenvolvidos de modo a aprovar a instalação de projetos em locais inadequados visando prevalecer, por exemplo, os aspectos exclusivamente econômicos e deixando de lado o propósito da avaliação de impacto ambiental (ALMEIDA, 2013).

$O$ estudo de alternativas garante à AIA significado importante, pois permite um questionamento criativo dos projetos que pelos meios tradicionais sequer seriam considerados (Sánchez, 2008), sendo considerado como um dos Princípios Operacionais da AIA (IAIA, 1999). Reforçando a importância da discussão de alternativas, Paliwal (2006) afirma que a sua proposição é uma das melhores práticas da AIA. Ainda, a própria legislação brasileira exige que sejam consideradas todas as alternativas locacionais do projeto dentro do Estudo de Impacto Ambiental (EIA) (CONAMA, 1986).

De acordo com a tipologia de um projeto, a discussão de alternativas locacionais torna-se ainda mais relevante (FERNANDES et al., 2017a), o que se aplica ao caso de aterros sanitários. Aterro sanitário consiste na técnica de disposição de resíduos sólidos urbanos no solo que utiliza princípios de engenharia para confinar os resíduos sólidos à menor área possível e reduzi-los ao menor volume permissível, cobrindo-os com uma camada de terra na conclusão de cada jornada de trabalho ou intervalos menores, se necessário, sem causar danos ou riscos à saúde pública e à segurança, minimizando os 
impactos ambientais (ABNT, 1992). Ainda, é um método de disposição dos resíduos sólidos que demanda estudos e ações para a correta disposição final desses detritos (DALMAS et al., 2011), consideradas as técnicas para a acomodação dessas substâncias, bem como 0 local apropriado para se implantar um empreendimento desse porte (CRESPO, 2006). Dessa forma, para o caso de um aterro sanitário, o atendimento a esses critérios significa avaliar todo o território disponível quanto à existência de sítios aptos a acomodar tal atividade. $O$ emprego íntegro dessa ferramenta de estudo de alternativas rege o conjunto de elementos fundamentais para a determinação da viabilidade ambiental do empreendimento (MONTAÑO et al., 2012).

Além disso, a que se considerar que o aumento do processo de urbanização reduz as áreas possivelmente adequadas para implantação de aterros sanitários, o que faz com que o estudo locacional se torne cada vez mais necessário, principalmente, nas Regiões Sudeste e Sul do Brasil, onde se aumenta cada vez mais o número de aterros sanitários construídos (MORAIS, 2005); e que as melhores alternativas para a localização de aterros são as que consideram os aspectos técnicos, ambientais e socioeconômicos com o propósito de reduzirem os impactos negativos ao meio ambiente originados por esse tipo de empreendimento (CALIJURI et al., 2002).

Dada a relevância desse contexto, torna-se pertinente o objetivo do presente trabalho de avaliar como estão sendo contemplados os estudos de alternativas locacionais para construção de aterros sanitários dentro dos processos de licenciamento com Avaliação de Impacto Ambiental no Estado de Minas Gerais, focando em descrever a metodologia empregada e em levantar os critérios utilizados para a seleção de áreas para a construção desta tipologia de empreendimento.

\section{METODOLOGIA}

O procedimento metodológico do presente trabalho consistiu na análise do conteúdo de documentos. No caso, os documentos foram os Estudos de Impacto Ambiental (EIA) voltados para a construção de aterros sanitários em processos de licenciamento ambiental estadual em Minas Gerais. As informações de interesse averiguadas nos EIAs foram as que dizem respeito ao estudo de alternativas locacionais dos mesmos. Assim, o presente trabalho se desenvolveu a partir das etapas de seleção e análise dos estudos de caso que contemplavam a aplicação dessas alternativas locacionais e comparação dos resultados encontrados com os critérios para seleção de áreas de aterro sugeridos pela literatura. 
A seleção dos estudos de caso foi realizada de forma on-line por meio do website da Secretaria de Estado de Meio Ambiente de Minas Gerais (SEMAD), onde é possível acessar o Sistema Integrado de Informação Ambiental (SIAM), que disponibiliza digitalmente documentos para consulta dos processos de licenciamento ambiental em Minas Gerais.O filtro para a pesquisa na plataforma do SIAM foi o código E-03-07-7 que, de acordo com a Deliberação Normativa (DN) no 74 de 2004 do Conselho Estadual de Meio Ambiente de Minas Gerais (COPAM), corresponde à atividade de "Tratamento e/ou Disposição Final de Resíduos Sólidos Urbanos”. A partir de 2018, esta DN foi substituída pela DN Copam ํㅜㄴ 217 de 2017, mas à época da realização da pesquisa, poucos processos tinham sido instituídos com base na nova norma legal, sendo utilizado apenas o contexto da primeira.

O resultado da busca no SIAM, realizada no período que compreende o período de 17 até 26 de maio de 2018, contabilizou um total de 1584 registros para a atividade em questão, sendo que 17 continham o EIA, mas apenas 8 apresentaram o documento digitalizado na plataforma, possibilitando seu acesso. Vale salientar que possivelmente nem todos os processos de licenciamento ambiental em Minas Gerais estão devidamente disponibilizados no SIAM, principalmente os processos mais antigos, ocorrendo também a hipótese de que nem todos os documentos dos processos de licenciamento estejam atualizados. Embora utilizar o SIAM possa ser um limite da pesquisa, essa só pôde ser realizada pela facilidade de seleção e acesso aos estudos de caso a partir da plataforma on-line, sendo que os dados encontrados permitiram atingir ao objetivo da pesquisa.

Por fim, buscou-se analisar dentre esses 8 documentos encontrados aqueles em que o Estudo de Impacto Ambiental (EIA) contou com critérios para o estudo de alternativas locacionais que, posteriormente, foram comparados com critérios apresentados e levantados na literatura.

A análise de cada estudo de caso foi realizada a partir da leitura dos EIAs disponibilizados. O enfoque foi dado para analisar os itens em que os estudos de alternativas locacionais estavam presentes. Dessa forma, foi possível ter uma visão geral do objeto de cada EIA, listando: o tipo de empreendimento e qual a empresa responsável por sua execução, além de se investigar como cada município realizou a pré-seleção de áreas, adotou os seus critérios de seleção e, por fim, comparou as opções de áreas e as selecionou. Essas informações foram sintetizadas e os critérios foram organizados para cada estudo de caso. 
Finalizando a análise, os critérios de seleção de áreas encontrados nos EIAs foram confrontados com a literatura. Para o levantamento dos critérios presentes na literatura foram usados os trabalhos de: Massunari (2000), Silva (2011), Montaño et al. (2012), Rezende et al. (2015), Fernandes et al. (2017b) e Carrilho et al. (2018). Além da NBR 13896/1997 que fixa as condições mínimas exigíveis para projeto, implantação e operação de aterros de resíduos não perigosos (ABNT, 1997); do Guia de Elaboração de Projetos de Aterros Sanitários para Resíduos Sólidos Urbanos, elaborado pelo Conselho Regional de Engenharia e Agronomia do Paraná (CREA-PR, 2009); e da Diretriz Técnica nº 04 de 2017 da Fundação Estadual de Proteção Ambiental Henrique Luís Roessler (FEPAM, 2017), órgão responsável pelo licenciamento ambiental do estado do Rio Grande do Sul.

\section{RESULTADOS E DISCUSSÃO}

Os municípios mineiros com processo de licenciamento ambiental que apresentaram Estudo de Impacto Ambiental (EIA) referente à atividade de "Tratamento e/ou Disposição Final de Resíduos Sólidos Urbanos" disponíveis para download na plataforma do SIAM durante o período de pesquisa são mostrados na Tabela 1.

Além desses 8 processos, foram encontrados outros 9 empreendimentos que indicavam registros de seus respectivos EIAs no website do SIAM para a atividade de "Tratamento e/ou Disposição Final de Resíduos Sólidos Urbanos" no âmbito da DN Copam no 74/2004, porém não foi possível o acesso desses documentos, pois eles não se encontravam digitalizados. Esses municípios e as respectivas numerações dos seus processos técnicos eram: Alfenas (Processo Técnico: 00451/1995), Divinópolis (37443/2013), Governador Valadares (16001/2014), Itaúna (00399/1998), João Monlevade (14037/2011), Juiz de Fora (01276/2007), Leopoldina (30234/2012), Mariana (00184/2000) e Uberaba (08233/2011). No caso de Uberaba, havia dois processos com EIAs relacionados, mas somente um disponível e que foi avaliado. 
Tabela 1 - Processos mineiros de licenciamento de aterros sanitários com EIA disponível para download no SIAM

\begin{tabular}{|c|c|c|c|}
\hline $\begin{array}{l}\text { Município do } \\
\text { empreendimento }\end{array}$ & $\begin{array}{l}\text { Processo } \\
\text { Técnico }\end{array}$ & Empreendimento & $\begin{array}{c}\text { Entidade } \\
\text { elaboradora/Ano }\end{array}$ \\
\hline Betim & $01034 / 2005$ & $\begin{array}{c}\text { Central de Tratamento de Resíduos de } \\
\text { Betim/MG }\end{array}$ & Essencis/2012 \\
\hline Betim & $16590 / 2008$ & $\begin{array}{c}\text { Aterro Sanitário e Unidade de Compostagem } \\
\text { de Betim/MG }\end{array}$ & Viasolo/2009 \\
\hline $\begin{array}{l}\text { Governador } \\
\text { Valadares }\end{array}$ & $17867 / 2009$ & $\begin{array}{c}\text { Sistema de Tratamento e Disposição Final de } \\
\text { Resíduos Sólidos Urbanos de Governador } \\
\text { Valadares/MG }\end{array}$ & Escoar/2003 \\
\hline Guaranésia & $20615 / 2005$ & $\begin{array}{c}\text { Consórcio Intermunicipal de Desenvolvimento } \\
\text { Sócio-Econômico e Ambiental de Municípios } \\
\text { da Região da AMOG }\end{array}$ & AMOG/2006 \\
\hline Montes Claros & $11771 / 2011$ & $\begin{array}{c}\text { Central de Tratamento de Resíduos Sólidos } \\
\text { Montes Claros/MG }\end{array}$ & Viasolo/2011 \\
\hline Ponte Nova & $07140 / 2013$ & Aterro Sanitário Municipal de Ponte Nova/MG & CISAB/2014 \\
\hline$U$ & 1 & $\begin{array}{c}\text { Tratamento e Disposição de resíduos não- } \\
\text { perigosos de Uberaba/MG }\end{array}$ & Soma/2012 \\
\hline Uberlândia & $00353 / 1996$ & Aterro Sanitário de Uberlândia/MG & Limpebras/2009 \\
\hline
\end{tabular}

Fonte: Elaborado pelos autores

O Processo Técnico 01034/2005 do município de Betim para o licenciamento da Central de Tratamento de Resíduos de Betim, visava à regularização ambiental da área já implantada no município na forma de solicitação de Licença de Operação de caráter corretivo do empreendimento. Sob esta justificativa, o EIA (ESSENCIS, 2012) não apresentou estudo de alternativas para seleção da área. Assim, entre os 8 municípios com EIAs disponibilizados no SIAM, 7 apresentavam estudo de alternativas locacionais e foram considerados na presente pesquisa.

Os estudos de alternativas locacionais para a implementação de aterro sanitários presentes no EIA devem ser elaborados de acordo a Resolução CONAMA nํ 001, de 23 de janeiro de 1986 e seguir suas orientações que estabelecem a obrigatoriedade de se contemplar todas as possíveis alternativas de localização do projeto, incluindo a hipótese de sua não execução (CONAMA, 1986). É por consequência dessa diretriz que os estudos de alternativas locacionais devem se esforçar na tentativa de propor e avaliar todas as áreas possíveis de uma região para construção do aterro sanitário.

Os processos analisados realizaram o estudo de alternativas de maneira particular, impondo critérios que foram considerados pela equipe elaboradora do estudo como necessários para a seleção das áreas de disposição final de resíduos.

Na sequência, será apresentada uma breve descrição (em ordem alfabética pelo nome do município) de como foi realizada a seleção de áreas para a construção de aterros sanitários nos EIAs dos processos selecionados. Cabe ressaltar que o presente trabalho 
não teve o objetivo de avaliar a efetividade dos estudos de alternativas realizados nos estudos de caso selecionados, ficando isso como recomendação para estudos futuros. No entanto, ao final da descrição do processo de seleção de alternativas de cada caso, alguns pontos foram destacados.

\subsection{Betim}

O estudo de alternativas do EIA para a implantação de uma Central de Tratamento de Resíduos Sólidos no município de Betim foi realizado dispondo-se, segundo o documento, de uma avaliação técnica, econômica, política e social da adequabilidade das áreas para disposição final dos resíduos (VIASOLO, 2009). Foram avaliadas 17 áreas préselecionadas com potencialidade para instalação do aterro sanitário.

Inicialmente, foram estabelecidos aspectos gerais para a avaliação e seleção dessas áreas. A metodologia para a escolha se dividiu na pré-seleção de mapas e imagens de satélite, visita em campo de cada área e avaliação por meio de matriz específica. Os critérios de seleção ficaram divididos em restrições, no qual para cada item considerado apto (A) foi atribuído uma nota 1, parcialmente apto (B) 0,5 e não apto (C), nota 0. Após uma análise descritiva das características das 17 áreas, foi elaborada uma matriz com as principais qualidades ambientais de cada área e suas pontuações associadas. As áreas 12 , 16 e 17 apresentaram as maiores notas e, por isso, foram consideradas as mais adequadas para a implantação do aterro sanitário. No entanto, a consultoria considerou a área 17 a mais adequada por apresentar parâmetros considerados mais favoráveis a implantação da Central de Tratamento de Resíduos Sólidos de Betim.

A avaliação aqui realizada, e que foi realizada da mesma forma para o município de Montes Claros (mesma consultoria responsável pelos dois estudos), chama a atenção para a quantidade de áreas analisadas (17 em Betim e 11 em Montes Claros), prática que parece seguir a orientação da legislação de se contemplar todas as possíveis alternativas de localização do projeto (CONAMA, 1986).

\subsection{Governador Valadares}

Este processo refere-se ao Licenciamento Ambiental do Sistema de Tratamento e Disposição Final de Resíduos Sólidos Urbanos do Município de Governador Valadares. Ao iniciar a descrição de como foi realizada a análise das alternativas locacionais, o EIA 
menciona que o objetivo da escolha foi fundamentado em critérios técnicos que visam à menor degradação do meio ambiente (ESCOAR, 2003), sendo apresentados os aspectos que conduziram o projeto para seleção das áreas e os fatores priorizados na análise de alternativas locacionais. No EIA, foram avaliadas 3 áreas a partir desses aspectos e fatores prioritários. Optou-se pela escolha da área onde já se estabelecia o atual aterro da cidade; não houve ponderação dos critérios mencionados; e a escolha se norteou, segundo o EIA (ESCOAR, 2003), principalmente, pelo fato de que a área escolhida já vem sendo utilizada há 12 anos para disposição de resíduos e apresenta condições mais favoráveis para a qualidade ambiental necessária.

Neste caso, não foi apresentado o processo de seleção da alternativa e, aparentemente, as outras duas alternativas apresentadas seriam reconhecidamente inferiores à selecionada no EIA, deficiência dos EIAs já apontada por MPF (2004). Em outras palavras, parece que já estava certo a utilização da área atual e as demais foram propostas apenas como proforma.

\subsection{Guaranésia}

O estudo de alternativas para áreas de implantação de uma Central de Tratamento de Resíduo no município de Guaranésia presente no EIA analisado teve como finalidade a solução para o problema do tratamento e destino final dos resíduos sólidos urbanos dos municípios de Arceburgo, Cabo Verde, Guaranésia, Monte Belo, Muzambinho e São Pedro da União. Os representantes destes municípios se uniram e formaram o Consórcio Intermunicipal de Desenvolvimento Socioeconômico e Ambiental de Municípios da Região da Associação dos Municípios da Baixa Mogiana (AMOG).

O documento faz uma justificativa da seleção do local a ser implantado o aterro sanitário, enfatizando que a escolha foi realizada conforme critérios técnicos que visam minimizar o impacto negativo sobre o meio ambiente, fundamentados em estudos realizados por pesquisadores e profissionais que atuam na área, nas legislações vigentes para a adequação da atividade de disposição final de resíduos sólido e, "mais do que isso", em imposições de critérios restritivos - "profundidade do lençol freático, distância de corpo d’água, permeabilidade do solo etc" (AMOG, 2006).

Com base nessas indicações, foi elaborada uma planilha de viabilidade locacional onde foram atribuídos pesos e notas para os critérios. No entanto, foi avaliada apenas uma área pré-selecionada. Segundo o EIA (AMOG, 2006), os parâmetros para se estabelecer a 
aptidão preliminar de uma área adequada para implantação de um aterro sanitário deveriam somar, no mínimo, 70\% dos pontos da planilha e não apresentar valores restritivos (nota zero). No caso da área já pré-selecionada, os valores foram de $75 \%$ e nenhuma das notas foi nula, mostrando-se, assim, como uma área, preliminarmente, adequada. Apenas cerca de $30 \%$ da área estudada não deveria ser utilizada para a execução de aterros, por compreenderem um limite inferior a 200 metros de corpos d'água.

Assim, deve-se considerar que os critérios restritivos utilizados foram citados vagamente. $\mathrm{O}$ estudo de alternativas locacionais avaliou uma região e teve como resultado apenas uma análise preliminar de uma parcela desta área que estaria adequada para receber o empreendimento. Dessa forma, o estudo se limitou a apontar a porcentagem onde não deveria se executar o aterro por apresentar a restrição de uma distância menor do que 200 m de corpos d'água, e não realizou uma avaliação de onde potencialmente seria a melhor área para construção do aterro.

Assim, a análise de alternativas se restringiu à avaliação de uma única alternativa, impedindo-se o confronto de opções e configurando um reducionismo da aplicação da AIA, que se torna reativa ao se restringir à identificação de medidas mitigadoras (AGRA FILHO et al., 2012). Embora tenha existido um trecho do EIA dedicado ao estudo de alternativas, pode-se dizer que este caso reflete o que apontam estudos como os de Hickie e Wade (1998), Steinemann (2001), Pinho et al. (2007) e Kruopiene et al. (2009) de que a proposição de alternativas tecnológicas e locacionais é incipiente ou inexistente.

\subsection{Montes Claros}

O estudo de alternativas locacionais presente no EIA referente a implantação da Central de Tratamento de Resíduos Sólidos de Montes Claros visou uma avaliação técnica, econômica, política e social para escolha da melhor área a receber os resíduos sólidos do município. No EIA (VIASOLO, 2011), foram avaliadas 11 áreas que apresentavam potencialidade para a construção do aterro.

O EIA deste processo foi realizado pela mesma consultoria que elaborou o estudo de alternativas para o município de Betim. Sendo assim, a metodologia empregada foi a mesma: inicialmente, foram estabelecidos aspectos gerais para a avaliação e seleção dessas áreas. A metodologia para a escolha se dividiu na pré-seleção de mapas e imagens de satélite, visita em campo de cada área e avaliação por meio de matriz específica. Os critérios de seleção ficaram divididos em restrições, no qual para cada item considerado 
apto (A) foi atribuído uma nota 1 , parcialmente apto (B) 0,5 e não apto (C), nota 0 . Após uma análise descritiva das características das 11 áreas, foi elaborada uma matriz com as principais qualidades ambientais de cada área e suas pontuações associadas. As áreas 5, 6 e 11 apresentaram as maiores notas e, por isso, foram consideradas as mais adequadas para a implantação do aterro sanitário. Foi escolhida a área 6 como a mais adequada por apresentar parâmetros considerados mais favoráveis a implantação da Central de Tratamento de Resíduos Sólidos de Montes Claros. A consideração sobre este estudo foi realizada junto com o estudo do município de Betim.

\subsection{Ponte Nova}

O estudo de alternativa locacional foi destinado à implantação do novo aterro sanitário de Ponta Nova realizado pelo Consórcio de Saneamento Básico da Zona da Mata de Minas Gerais (CISAB), e sugere a averiguação de dois locais para a implantação do aterro, além de uma área proposta pela própria Secretaria Municipal de Meio Ambiente (SEMAM) da cidade. O estudo afirmou que a área proposta pela SEMAM foi o local escolhido para construção do aterro devido a ir de encontro com as exigências legais detalhadas no item "Análise comparativa entre as Alternativas Locacionais Propostas", porém esse item não estava presente no EIA em questão, que apresentava apenas informações da metodologia usada (CISAB, 2014).

Segundo o EIA (CISAB, 2014), a seleção de áreas ocorreu em duas etapas preliminares. A primeira correspondia à aplicação de critérios eliminatórios "embasados principalmente nas restrições impostas pela legislação ambiental” e a segunda etapa aplicou critérios seletivos segundo aspectos ambientais, técnicos e econômicos. Estes critérios foram avaliados por meio da combinação de pontuação e pesos de cada critério. Por último, o estudo descreveu que o local escolhido se mostrou adequado, apresentando baixos impactos, tanto em termos técnicos como ambientais, e indicou que a recomendação do local escolhido foi reforçada pela sua facilidade de acesso.

Assim, o estudo de alternativas de Ponte Nova citou os critérios restritivos utilizados, criou uma divisão de critérios seletivos que engloba de maneira clara os aspectos ambientais, técnicos e econômicos, além de atribuir uma variação de pesos na ponderação dos critérios apresentada. No entanto, não foi apresentado como foi realizada a análise comparativa dessas áreas; mesmo sendo mencionado, esse item ele não apareceu no documento. Aparentemente, a alternativa a ser implantada já havia sido escolhida e 0 
restante do estudo, conforme já apontava em outro contexto Zubair (2001), abrangeu alternativas ambientalmente inviáveis.

\subsection{Uberaba}

O estudo de alternativas locacionais contido no EIA foi desenvolvido para ampliação da capacidade de recebimento de resíduos sólidos e correta destinação desses resíduos no município. O estudo foi dividido em levantamento bibliográfico, legislativo e de dados espaciais disponíveis e avaliação de critérios para escolha das áreas e aprofundamento da investigação do local escolhido por meio das informações geradas (SOMA, 2012).

Inicialmente, considerou-se todo território da cidade, baseado em levantamento e mapeamentos oficiais, com o objetivo de limitar quais as áreas com uma aptidão prévia para uma análise futura mais detalhada. Assim, foram selecionadas 4 áreas para um estudo mais minucioso. A metodologia empregada na análise das alternativas locacionais considerou três grupos de critérios: ambientais (capacidade natural das áreas em atenuar os possíveis impactos ambientais negativos), engenharia (complexidade técnica envolvida e tempo incidente sobre as diversas fases do aterro sanitário) e econômico (principais custos variáveis com a escolha locacional relacionado às fases de implementação e operação dos aterros). Estes critérios foram avaliados por um sistema de notas e pesos, sendo atribuída uma nota final para cada alternativa. A área 4 foi a melhor avaliada e, portanto, escolhida.

Logo, o estudo para Uberaba, além de considerar aspectos gerais para seleção da região desejável de se implementar o aterro sanitário, organizou uma planilha que mostrou o modo de avaliação atribuído a cada critério selecionado. O estudo também justificou a escolha da área adequada por meio da indicação de suas principais vantagens e das principais desvantagens das áreas concorrentes. De forma superficial, o estudo parece não contemplar as principais falhas apontadas pela literatura.

\subsection{Uberlândia}

$\mathrm{Na}$ análise de alternativas locacionais foram indicadas e comparadas 4 áreas com potencial para implantação do aterro sanitário e elaboradas matrizes de escolha a partir da atribuição de pontuações para avaliar qual seria a melhor alternativa. O estudo sugeriu que a área selecionada seria aquela que apresentasse o melhor desempenho em termos 
ambientais (dos meios físico e biótico), técnicos, econômicos, sociais e legais para esta finalidade. Segundo o EIA (LIMPEBRAS, 2009), os aspectos sociais foram levados em conta assumindo um papel de relevantes, em que entrevistas foram realizadas com a população do entorno das possíveis áreas. A metodologia do trabalho iniciou-se com o levantamento dos aspectos de restrições para a instalação do aterro, onde algumas áreas foram descartadas. Após a definição dos aspectos de restrições, foram definidos os atributos fundamentais que auxiliariam na escolha da área mais adequada, critérios de prioridade e ponderação para os atributos definidos.

Neste ponto, a área 2 foi desclassificada pois não alcançou a pontuação mínima estabelecida (30 pontos). Assim, as áreas com maior potencial para implantação do aterro sanitário a partir dos critérios expostos e, portanto, com maiores pontuações foram as áreas 1, 2 e 4. Foi realizada, então, na finalização do estudo de alternativa, uma análise mais detalhada das áreas remanescentes, levando em consideração três grupos de critérios que vão além dos critérios já utilizados: critérios técnico-operacionais, critérios econômicofinanceiros e critérios político-sociais, atribuindo ponderações e pesos para cada um. Por fim, aplicando todos os critérios enunciados com seus devidos pesos, a área com maior pontuação foi a Área 4, sendo essa a escolhida como a melhor área para implementação do aterro sanitário. Esta área era justamente a proposta inicial da prefeitura.

A metodologia proposta é bastante interessante, pois houve, além dos aspectos restritivos, a imposição de critérios bem ponderados e, ainda mais, destacou-se a divisão de itens em critérios na aplicação de grupos bem distribuídos (Aplicação TécnicoOperacional, Aplicação Econômico-financeira e Aplicação Político-Social) que favorecem a visão ampla de interesses que o EIA deve ter (SÁNCHEZ, 2008). O documento, ainda, afirmou ter levado em consideração a opinião social por meio de entrevistas com a população, o que remete a uma boa prática, já que o envolvimento do público na tomada de decisão é um componente fundamental da AIA (HARTLEY e WOOD, 2005). No entanto, como a área escolhida foi o local previamente sugerido pela prefeitura, questiona-se se o processo não se encaminhou para uma seleção baseada no interesse de validar uma escolha já realizada.

\subsection{Síntese das metodologias de seleção utilizadas}

De modo geral e breve, as metodologias de seleção para a área de construção dos aterros presentes nos estudos de caso podem ser assim resumidas em: 
- utilização de critérios para comparação de áreas sem um processo estruturado (caso de Governador Valadares);

- aplicação de uma matriz de critérios com pesos e notas em um conjunto de áreas (caso de Guaranésia, mas onde foi avaliada apenas uma área);

- uso de uma metodologia estruturada e detalhada, onde inicialmente aplicam-se critérios gerais para a pré-seleção de áreas e as áreas pré-selecionadas são avaliadas por uma matriz de critérios com pesos e notas (casos de Betim, Montes Claros, Uberaba e Uberlândia. Parece ter sido a metodologia de Ponte Nova, mas o EIA não a apresentou em detalhes, apenas a citou).

\subsection{A literatura e os critérios recorrentes na condução dos estudos de alternativas locacionais}

Nos estudos de caso analisados, a composição de critérios de alguns estudos de alternativas foi detalhada e parece ter sido cuidadosamente elaborada, enquanto outros estudos foram mais simplificados. Somando-se os critérios apresentados pelos artigos, normas e guias para a seleção de área de aterros e os critérios apresentados pelos estudos de caso analisados, foi possível construir a Tabela 2. Nela, os critérios apontados pela literatura foram avaliados como presentes ou ausentes no estudo de caso, mas também é apresentada a informação caso algum critério esteja presente de modo geral no estudo de caso, ou seja, sem especificar o valor para o parâmetro detalhado pela literatura.

Tabela 2 - Comparação dos critérios de seleção de área de aterros levantados nos estudos de caso com a literatura

\begin{tabular}{|c|c|c|c|c|c|c|c|c|}
\hline Critérios da literatura & B & GV & G & MC & PN & Ub & UI & Trabalhos que utilizam os critérios \\
\hline $\begin{array}{c}\text { Declividade superior a 1\% } \\
\text { e inferior a 30\%. }\end{array}$ & $\checkmark$ & - & $\checkmark$ & $\checkmark$ & - & $x$ & - & $\begin{array}{c}\text { ABNT (1997); Massunari (2000); Rezende et } \\
\text { al. (2015) }\end{array}$ \\
\hline $\begin{array}{c}\text { Declividade superior a 2 e } \\
\text { inferior a 20\%. }\end{array}$ & $x$ & - & $\checkmark$ & $x$ & - & $x$ & - & $\begin{array}{c}\text { CREA-PR (2009); Silva (2011); Rezende et al. } \\
\text { (2015); Fernandes et al. (2017b) }\end{array}$ \\
\hline $\begin{array}{c}\text { Solo com coeficiente de } \\
\text { permeabilidade inferior a } \\
1 \times 10^{-6} \text { cm/s }\end{array}$ & $x$ & $\checkmark$ & - & $x$ & - & - & - & ABNT (1997); CREA-PR (2009) \\
\hline $\begin{array}{c}\text { Solo com zona não- } \\
\text { saturada de espessura } \\
\text { superior a 3m }\end{array}$ & $x$ & - & - & $x$ & $x$ & - & - & $\begin{array}{c}\text { ABNT (1997); Massunari (2000); CREA-PR } \\
\text { (2009) }\end{array}$ \\
\hline $\begin{array}{c}\text { Solo de materiais argiloso, } \\
\text { siltico argiloso ou areno } \\
\text { argiloso }\end{array}$ & $\checkmark$ & - & $\checkmark$ & $\checkmark$ & $x$ & $\checkmark$ & - & $\begin{array}{c}\text { Massunari (2000); CREA-PR (2009); Silva } \\
\text { (2011) }\end{array}$ \\
\hline $\begin{array}{c}\text { Distância mínima de 200m } \\
\text { das falhas geológicas }\end{array}$ & $x$ & $x$ & $x$ & $x$ & $x$ & $x$ & $x$ & Montaño et al. (2012); Fernandes et al. \\
(2017b)
\end{tabular}


Tabela 2 - Comparação dos critérios de seleção de área de aterros levantados nos estudos de caso com a literatura

\begin{tabular}{|c|c|c|c|c|c|c|c|c|}
\hline Critérios da literatura & B & GV & $\mathbf{G}$ & MC & PN & $\mathrm{Ub}$ & UI & Trabalhos que utilizam os critérios \\
\hline $\begin{array}{l}\text { Distância mínima de } 200 \mathrm{~m} \\
\text { de qualquer coleção } \\
\text { hídrica ou curso de água }\end{array}$ & - & - & $\checkmark$ & - & - & - & - & $\begin{array}{c}\text { ABNT (1997); Massunari (2000); CREA-PR } \\
\text { (2009); Silva (2011); Rezende et al. (2015); } \\
\text { Fernandes et al. (2017b) }\end{array}$ \\
\hline $\begin{array}{l}\text { Distância mínima de } 300 \mathrm{~m} \\
\text { de qualquer coleção } \\
\text { hídrica ou curso de água }\end{array}$ & - & - & $\checkmark$ & - & - & - & - & Rezende et al. (2015); Carrilho et al. (2018) \\
\hline $\begin{array}{l}\text { Distância do centro } \\
\text { gerador }\end{array}$ & $\checkmark$ & $\checkmark$ & $\checkmark$ & $\checkmark$ & $\checkmark$ & $\checkmark$ & $\checkmark$ & $\begin{array}{c}\text { Massunari (2000); FEPAM (2017); Carrilho et } \\
\text { al. (2018) }\end{array}$ \\
\hline $\begin{array}{c}\text { Distância mínima de } 500 \mathrm{~m} \\
\text { de núcleos } \\
\text { populacionais/área } \\
\text { urbanizada }\end{array}$ & $\checkmark$ & - & $\checkmark$ & $\checkmark$ & $\checkmark$ & - & $\checkmark$ & $\begin{array}{c}\text { ABNT (1997); Massunari (2000); CREA-PR } \\
\text { (2009); Rezende et al. (2015); Fernandes et } \\
\text { al. (2017b). }\end{array}$ \\
\hline $\begin{array}{l}\text { Distância mínima de } 2 \mathrm{~km} \\
\text { de zonas residenciais }\end{array}$ & $x$ & $x$ & $\checkmark$ & $x$ & $x$ & $x$ & $x$ & $\begin{array}{l}\text { CREA-PR (2009); Silva (2011); Rezende et al. } \\
\text { (2015); Carrilho et al. (2018). }\end{array}$ \\
\hline $\begin{array}{c}\text { Distância mínima de } 20 \mathrm{~m} \\
\text { de rodovias }\end{array}$ & $x$ & $x$ & $x$ & $x$ & $x$ & - & $\checkmark$ & CREA-PR (2009) \\
\hline $\begin{array}{l}\text { Distância mínima de } 50 \mathrm{~m} \\
\text { de rodovias }\end{array}$ & $x$ & $x$ & $x$ & $x$ & $x$ & - & $\checkmark$ & Silva (2011) \\
\hline $\begin{array}{c}\text { Distância de } \\
\text { aeroportos/aeródromos }\end{array}$ & $x$ & $x$ & $x$ & $\checkmark$ & $\checkmark$ & $x$ & $\checkmark$ & CREA-PR (2009); Montaño et al. (2012) \\
\hline $\begin{array}{l}\text { Profundidade do lençol } \\
\text { freático }>2 \mathrm{~m}\end{array}$ & $x$ & - & $\checkmark$ & $x$ & $\checkmark$ & - & $x$ & CREA-PR (2009) \\
\hline $\begin{array}{l}\text { Profundidade do lençol } \\
\text { freático }>1,5 \mathrm{~m}\end{array}$ & $x$ & - & $\checkmark$ & $x$ & $\checkmark$ & - & $x$ & Massunari (2000) \\
\hline $\begin{array}{l}\text { Composição da cobertura } \\
\text { vegetal }\end{array}$ & $\checkmark$ & $x$ & $x$ & $\checkmark$ & $\checkmark$ & $x$ & $x$ & $\begin{array}{c}\text { ABNT (1997); CREA-PR (2009); Massunari } \\
\text { (2000); Montaño et al. (2012); Rezende et al. } \\
\text { (2015); FEPAM (2017) }\end{array}$ \\
\hline Áreas sujeitas à inundação & $x$ & $x$ & $x$ & $x$ & $x$ & $x$ & $x$ & ABNT (1997); CREA-PR (2009) \\
\hline Condições de acessos & $\checkmark$ & $\checkmark$ & $\checkmark$ & $\checkmark$ & $\checkmark$ & $\checkmark$ & $\checkmark$ & $\begin{array}{c}\text { ABNT (1997); CREA-PR (2009); Massunari } \\
\text { (2000); Rezende et al. (2015); FEPAM (2017); } \\
\text { Fernandes et al. (2017b) }\end{array}$ \\
\hline $\begin{array}{l}\text { Tamanho disponível e vida } \\
\text { útil }\end{array}$ & $\checkmark$ & $\checkmark$ & $x$ & $\checkmark$ & $\checkmark$ & $\checkmark$ & $\checkmark$ & $\begin{array}{l}\text { ABNT (1997); Massunari (2000); CREA-PR } \\
\text { (2009); FEPAM (2017); Carrilho et al. (2018) }\end{array}$ \\
\hline $\begin{array}{c}\text { Disponibilidade de material } \\
\text { de empréstimo para } \\
\text { cobertura }\end{array}$ & $\checkmark$ & $\checkmark$ & $x$ & $\checkmark$ & $\checkmark$ & $\checkmark$ & $\checkmark$ & Massunari (2000); CREA-PR (2009) \\
\hline $\begin{array}{l}\text { Identificação de áreas } \\
\text { protegidas }\end{array}$ & $x$ & $x$ & $x$ & $x$ & $\checkmark$ & $x$ & $\checkmark$ & $\begin{array}{c}\text { Massunari (2000); CREA-PR (2009); Silva } \\
\text { (2011); Montaño et al. (2012); Rezende et al. } \\
\text { (2015) }\end{array}$ \\
\hline $\begin{array}{l}\text { Direção dos ventos } \\
\text { predominantes }\end{array}$ & $x$ & $x$ & $x$ & $x$ & $\checkmark$ & $x$ & $x$ & $\begin{array}{c}\text { CREA-PR (2009); Montaño et al. (2012); } \\
\text { FEPAM (2017) }\end{array}$ \\
\hline $\begin{array}{l}\text { Existência de barreiras à } \\
\text { propagação da poluição }\end{array}$ & $x$ & $x$ & $x$ & $x$ & $x$ & $x$ & $x$ & FEPAM (2017) \\
\hline $\begin{array}{l}\text { Atenuação do impacto } \\
\text { visual negativo }\end{array}$ & $x$ & $x$ & $x$ & $x$ & $\checkmark$ & $x$ & $x$ & ABNT (1997); FEPAM (2017) \\
\hline $\begin{array}{c}\text { Influência sobre as } \\
\text { atividades e características } \\
\text { do município }\end{array}$ & $x$ & $x$ & $x$ & $x$ & $\checkmark$ & $\checkmark$ & $\checkmark$ & FEPAM (2017) \\
\hline $\begin{array}{c}\text { Realizar levantamento de } \\
\text { inventário (banco de } \\
\text { dados) }\end{array}$ & $x$ & $\checkmark$ & $x$ & $x$ & $x$ & $x$ & $x$ & CREA-PR (2009) \\
\hline $\begin{array}{l}\text { Compatibilidade com uso } \\
\text { e ocupação do solo do } \\
\text { município }\end{array}$ & $\checkmark$ & $\checkmark$ & $\checkmark$ & $\checkmark$ & $\checkmark$ & $\checkmark$ & $\checkmark$ & $\begin{array}{c}\text { ABNT (1997); Massunari (2000); CREA-PR } \\
\text { (2009); Montaño et al. (2012); Rezende et al. } \\
\text { (2015) }\end{array}$ \\
\hline $\begin{array}{l}\text { Considerar preservação } \\
\text { dos biomas }\end{array}$ & $x$ & $x$ & $x$ & $x$ & $x$ & $x$ & $x$ & CREA-PR (2009) \\
\hline $\begin{array}{l}\text { Considerar a existência de } \\
\text { espécies da flora } \\
\text { ameaçada de extinção }\end{array}$ & $x$ & $x$ & $x$ & $x$ & $x$ & $x$ & $x$ & CREA-PR (2009) \\
\hline
\end{tabular}


Tabela 2 - Comparação dos critérios de seleção de área de aterros levantados nos estudos de caso com a literatura

\begin{tabular}{|c|c|c|c|c|c|c|c|c|}
\hline Critérios da literatura & B & GV & $\mathbf{G}$ & MC & PN & Ub & UI & Trabalhos que utilizam os critérios \\
\hline $\begin{array}{c}\text { Considerar a existência de } \\
\text { espécies da fauna } \\
\text { ameaçada de extinção }\end{array}$ & $x$ & $x$ & $x$ & $x$ & $x$ & $x$ & $x$ & CREA-PR (2009) \\
\hline $\begin{array}{l}\text { Considerar a existência de } \\
\text { sítios paleontológicos }\end{array}$ & $x$ & $x$ & $x$ & $x$ & $x$ & $x$ & $x$ & CREA-PR (2009) \\
\hline $\begin{array}{c}\text { Considerar a existência de } \\
\text { bens culturais e } \\
\text { arqueológicos }\end{array}$ & $x$ & $x$ & $x$ & $x$ & $x$ & $x$ & $x$ & CREA-PR (2009) \\
\hline $\begin{array}{l}\text { Custos com a aquisição do } \\
\text { terreno }\end{array}$ & $\checkmark$ & $x$ & $\checkmark$ & $\checkmark$ & $\checkmark$ & $x$ & $\checkmark$ & ABNT (1997); CREA-PR (2009) \\
\hline $\begin{array}{c}\text { Custos para adequação do } \\
\text { local }\end{array}$ & $x$ & $x$ & $\checkmark$ & $x$ & $x$ & $x$ & $\checkmark$ & ABNT (1997); CREA-PR (2009) \\
\hline $\begin{array}{l}\text { Custos com material de } \\
\text { cobertura }\end{array}$ & $x$ & $x$ & $x$ & $x$ & $x$ & $x$ & $x$ & ABNT (1997); CREA-PR (2009) \\
\hline Custos com o transporte & $x$ & $x$ & $x$ & $x$ & $x$ & $x$ & $x$ & ABNT (1997); CREA-PR (2009) \\
\hline
\end{tabular}

$\checkmark$ Critério presente no estudo de caso.

$X$ Critério ausente no estudo de caso;

- Critério presente de modo geral no estudo de caso, ou seja, sem especificar valor para o parâmetro.

B - Betim; GV - Governador Valadares; G - Guaranésia; MC - Montes Claros; PN - Ponte Nova; Ub Uberaba; Ul - Uberlândia.

Fonte: Elaborado pelos autores

De acordo com os critérios apresentados na Tabela 2, percebe-se que aqueles remontados pela literatura foram mais detalhados que os utilizados pelos estudos de caso, ao apresentar valores de referência. Por exemplo, o critério para distância mínima de qualquer corpo hídrico ou curso d'água foi definido, na literatura, com distâncias mínimas de 200 ou 300 metros, porém apenas o município de Guaranésia considerou, explicitamente, estes valores (atribuindo pontuações diferentes para estas distâncias), enquanto que todos os outros municípios apenas consideraram que deve haver uma distância mínima desses recursos hídricos sem especificar qual seria a distância mínima necessária.

Os critérios da literatura presentes em todos os estudos de caso, mesmo que sem definição do valor do parâmetro, foram: caracterização do solo (incluindo, no mínimo, uma característica estudada: permeabilidade, espessura da camada e material de composição argiloso, síltico argiloso ou areno argiloso); distância de recurso hídrico; distância do centro gerador; distância de núcleos populacionais; condições de acesso à área; e compatibilidade com uso e ocupação do solo.

Alguns critérios da literatura não estiveram presentes em todos os estudos de casos, mas foram também bastante recorrentes, como a declividade, a disponibilidade de material de empréstimo para cobertura, o tamanho e a vida útil do aterro. Ainda, mesmo que menos frequentes que os anteriores, apareceram entre os critérios da literatura utilizados nos estudos de caso: custos com a aquisição do terreno; profundidade do lençol freático; 
distância de aeroportos/aeródromos; composição da cobertura vegetal; influência sobre as atividades e características do município; distância mínima de rodovias; identificação de áreas protegidas; e custos para adequação do local.

Os critérios da literatura que apareceram apenas uma vez nos estudos de caso foram: distância mínima de $2 \mathrm{~km}$ de zonas residenciais; direção dos ventos predominantes; atenuação do impacto visual negativo; e levantamento de inventário.

Por fim, alguns critérios presentes na literatura não foram acatados em nenhum estudo de alternativas locacionais dos casos analisados: o critério de existência de barreiras para a propagação da poluição proposto pelo Guia de Elaboração de Projetos de Aterros Sanitários para Resíduos Sólidos Urbanos do CREA-PR (CREA-PR, 2009); a distância mínima de 200m das falhas geológicas apresentada pelo trabalho de Fernandes et al. (2017b); a consideração técnica de não instalação de aterros em áreas sujeitas à inundação, bem como considerar a preservação dos biomas, conforme NBR 13896 (ABNT, 1997); e considerar a existência de espécies da flora e da fauna ameaçadas de extinção, de sítios paleontológicos, de bens culturais e arqueológicos e os custos com material de cobertura e com o transporte de resíduos, conforme Diretriz Técnica ${ }^{\circ} 04$ da Fundação Estadual de Proteção Ambiental Henrique Luís Roessler (FEPAM, 2017).

Em relação à quantidade de critérios presentes nos estudos de caso de modo geral (apenas citando o critério sem especificar o parâmetro limite que defina a escolha da área como é feito na literatura), Uberlândia e Ponte Nova apresentaram o maior número de critérios em conformidade com a literatura, seguidos de Governador Valadares, Uberaba, Guaranésia, Montes Claros e Betim, respectivamente. Porém, se forem considerados os critérios da literatura exatamente como eles estão sugeridos (critério e valor do parâmetro), Ponte Nova, seguida de Uberlândia e Guaranésia, são os municípios que apresentaram o maior número de critérios. Isso ocorre, pois, a sequência da ponderação logo após a apresentação dos critérios introdutórios nos EIAs desses municípios é mais bem detalhada do que os das outras cidades, abrangendo maior quantidade daqueles especificamente caracterizados na literatura. Nas demais cidades, os critérios aparecem sem 0 detalhamento de valores.

Uma vez que a dedicação em se estabelecer critérios para seleção de melhores áreas à implantação de um aterro sanitário presume o aumento do nível de conhecimento do meio ambiente a ser escolhido, o potencial causador de impacto do empreendimento no meio, segundo Sánchez (2008), é reduzido, colaborando com o propósito exigido na elaboração do estudo de alternativa locacional dentro do EIA. No entanto, verificou-se, em 
alguns estudos de caso, escassez de uma série de critérios recomendados pela literatura e, até mesmo, de obrigações especificadas em normas técnicas. Para estes casos e aqueles que restringiram a sua avaliação a poucos critérios, corre-se o risco de acontecer o apontado por Montaño et al. (2012), onde se descartam elementos fundamentais para a determinação da viabilidade ambiental do empreendimento.

\section{CONSIDERAÇÕES FINAIS}

De acordo com a disponibilidade de documentos dos processos de licenciamento, este estudo analisou 7 estudos de caso. Finalizando e resumindo os resultados encontrados, embora existissem similaridades procedimental e de critérios, não existiu um padrão de estudo de alternativas para a seleção de área para a implantação de aterros sanitários, nem na literatura e nem nos estudos de casos analisados. Isto, por um lado, pode favorecer a adaptação da metodologia escolhida ao caso em que está sendo aplicado; e, por outro lado, pode levar a manipulação de dados para que a área escolhida seja a desejada pelo empreendedor e não necessariamente a mais adequada do ponto de vista ambiental.

Assim, recomenda-se que estudos futuros sejam realizados tanto para analisar a efetividade dos estudos de alternativas locacionais realizados nos EIAs como para avaliar a pertinência da proposição de uma metodologia padrão para a seleção da área mais adequada para a implantação de um aterro sanitário. Os resultados destes estudos podem nortear a elaboração de Termos de Referências para os EIAs, a fim de guiar o estudo de alternativas locacionais de aterros sanitários, e a exigência por parte do órgão ambiental da obrigatoriedade do cumprimento de um conjunto de procedimentos mínimos a serem seguidos.

\section{REFERÊNCIAS}

ABNT - Associação Brasileira de Normas Técnicas. NBR 8419: Apresentação de projetos de aterros sanitários de resíduos sólidos urbanos. Rio de Janeiro: ABNT, 1992.

ABNT - Associação Brasileira de Normas Técnicas. NBR 13896: Aterro de resíduos não perigosos - Critérios para projeto, implantação e operação. Rio de Janeiro: ABNT, 1997.

AGRA FILHO, S. S.; MARINHO, M. M. O.; ORRICO, S. R. M.; SANTOS, F. C. Avaliação de Impactos Ambientais: uma discussão dos procedimentos metodológicos da aplicação no estado da Bahia. In: CONGRESSO BRASILEIRO DE AVALIAÇÃO DE IMPACTO, 1., 2012. [Anais...] São Paulo, 2012. 
ALMEIDA, M. R. R. Aplicação da abordagem sistêmica para análise da efetividade da Avaliação de Impacto Ambiental no Brasil: um estudo para os estados de São Paulo e Minas Gerais. 2013. 174 p. Tese (Doutorado em Ciências da Engenharia Ambiental). Escola de Engenharia de São Carlos, Universidade de São Paulo.

ALMEIDA, M. R. R.; MONTAÑO, M. Benchmarking na avaliação de impacto ambiental: o sistema mineiro frente às melhores práticas internacionais. Sociedade \& Natureza, v. 27, n. 1, p.81-96, 2015.

AMOG - Consórcio Intermunicipal de Desenvolvimento Socioeconômico e Ambiental de Municípios da Região da Associação dos Municípios da Baixa Mogiana. Estudo de Impacto Ambiental - Central de Tratamento de Resíduos de Guaranésia, 2006.

CALIJURI, M.; MELO, A. L. O.; LORENTZ, J. F. Identificação de Áreas para a Implantação de Aterros Sanitários com Uso de Análise Estratégica de Decisão. Informática Pública, v. 4, n.2, p.231-250, 2002.

CARRILHO, A. N.; CANDIDO, H. G.; SOUZA, A. D. Geoprocessamento aplicado na seleção de áreas para a implantação de aterro sanitário no município de Conceição das Alagoas (MG). Engenharia sanitária e ambiental, v. 23, n. 1, p. 201-206, 2018.

CISAB - Consórcio de Saneamento Básico da Zona da Mata de Minas Gerais. EIA-RIMA Aterro Sanitário Municipal de Ponte Nova/MG. 2014.

CONAMA - Conselho Nacional do Meio Ambiente (Brasil). Resolução n001, de 23 de janeiro de 1986. DOU, Brasília, 17 fev. 1986.

CREA-PR - Conselho Regional de Engenharia e Agronomia do Paraná. Guia de Elaboração de Projetos de Aterros Sanitários para Resíduos Sólidos Urbanos. 2009. Disponível em: https://www.crea-pr.org.br/ws/wp-content/uploads/2016/12/Publica\%C3\%A7\%C3\%B5esTem\%C3\%A1ticas-Guia-para-Elabora\%C3\%A7\%C3\%A3o-de-Projetos-de-AterrosSanit\%C3\%A1rios-para-Res\%C3\%ADduos-S\%C3\%B3lidos-Urbanos-Volume-ll.pdf. Acesso em: 02 nov. 2018.

CRESPO, D. P. Estudo de viabilidade ambiental para implantação do Aterro Sanitário de Canaã dos Carajás. 2006. 39p. Monografia (Especialização). Instituto de Geociências Universidade Federal de Minas Gerais, Belo Horizonte, 2006.

DALMAS, F. B.; GOVEIRA, S. S.; OLIVEIRA, F. R.; AMARAL, C. H.; MACEDO, A. B. Geoprocessamento aplicado à gestão de resíduos sólidos na UGRHI-11 - Ribeira de Iguape e Litoral Sul. Geociências, São Paulo, v. 30, n. 2, p. 285-299, 2011.

DUARTE, C. G.; DIBO, A. P. A.; SÁNCHEZ, L. E. O que diz a pesquisa acadêmica sobre avaliação de impacto e licenciamento ambiental no Brasil? Ambiente \& Sociedade, v. 20, n.1, p.245-278, 2017.

ESCOAR. Estudo de impacto ambiental: sistema de tratamento e disposição final de resíduos sólidos urbanos de Governador Valadares/MG.2003.

ESSENCIS. Estudo de impacto ambiental: central de tratamento de resíduos de Betim/MG.2012.

FEPAM - Fundação Estadual de Proteção Ambiental Henrique Luís Roessler. Diretriz Técnica n 04 de 2017. 2017. Disponível em: http://www.fepam.rs.gov.br/CENTRAL/DIRETRIZES/DT-0042017.PDF. Acesso em: 02 nov. 2018. 
FERNANDES, A. H. V.; CASSIANO, M. A.; GUIMARÃES, T. C. S.; ALMEIDA, M. R. R. Alternativas locacionais em avaliação de impacto ambiental de rodovias mineiras.

Desenvolvimento e Meio Ambiente, v. 43, p.73-90, 2017a.

FERNANDES, R. T. V; SILVEIRA, B. D. A.; OLIVEIRA, M. R. Planejamento urbano "open source": Um estudo de caso na identificação de áreas para implantação de aterro sanitário. HOLOS, v.8, p.126-144, 2017b.

HARTLEY, N.; WOOD, C. Public participation in environmental impact assessment: implementing the Aarhus Convention. Environmental Impact Assessment Review, v. 25, p.319-340, 2005.

HICKIE, D.; WADE, M. Development of guidelines for improving the effectiveness of environmental assessment. Environmental Impact Assessment Review, v.18, p.267-287, 1998.

IAIA - International Association for Impact Assessment. Principles of environmental impact assessment best practice. Fargo: IAIA, v.1, 1999.Disponível em:

http://www.iaia.org/publicdocuments/specialpublications/Principles\%200f\%20lA web.pdf. Acesso em: 02 nov. 2018.

KRUOPIENĖ, J.; ŽIDONIENĖ, S.; DVARIONIENĖ, J. Current practice and shortcomings of EIA in Lithuania. Impact Assessment and Project Appraisal, v.29, p.305-309, 2009.

LIMPEBRAS. Estudo de impacto ambiental: ampliação do aterro sanitário de Uberlândia/MG. 2009.

MASSUNARI, I. S. Pesquisa e seleção de áreas para aterro sanitário. Revista Limpeza Pública, Acervo digital, p.17-22, 2000.

MONTAÑO, M.; RANIERI, V. E. L.; SCHALCH, V; FONTES, A. T; CASTRO, M. C. A. A.; SOUZA, M. P. Integração de critérios técnicos, ambientais e sociais em estudos de alternativas locacionais para implantação de aterro sanitário. Engenharia Sanitária e Ambiental, v.17, n.1, p. 61-70, 2012.

MORAIS, J. L. Estudo da potencialidade de processos oxidativos avançados, isolados e integrados com processos biológicos tradicionais, para tratamento de chorume de aterro sanitário. 2005, 207p. Tese (Doutorado em Química). Universidade Federal do Paraná.

MORGAN, R. K. Environmental impact assessment: the state of the art. Impact Assessment and Project Appraisal, v.30, n.1, p.5-14, 2012.

MPF - Ministério Público Federal. Deficiências em estudos de impacto ambiental: síntese de uma experiência. Brasília: 4aㅡ Câmara de Coordenação e Revisão, Escola Superior do Ministério Público da União, 2004.

PALIWAL, R. EIA practice in India and its evaluation using SWOT analysis. Environmental Impact Assessment Review, v.26, p.492-510, 2006.

PINHO, P.; MAIA, R.; MONTERROSO, A. The quality of Portuguese Environmental Impact Studies: the case of small hydropower projects. Environmental Impact Assessment Review, v.27, p.189-205, 2007.

POPE, J.; BOND, A.; MORRISON-SAUNDERS, A.; RETIEF, F. Advancing the theory and practice of impact assessment: Setting the research agenda. Environmental Impact Assessment

Review, v.41, p.1-9, 2013. 
REZENDE, F. S.; CARRIELLO, F.; LEITE, M. B. A. Áreas potenciais para implantação de aterro sanitário em Ilha Grande-RJ. CEP, v. 24, p.340, 2015.

SÁNCHEZ, L. E. Avaliação de impacto ambiental: conceitos e métodos. São Paulo: Oficina de Textos, 2008. 495 p.

SILVA, C. R. Áreas potenciais para o aterro de resíduos sólidos industriais classe II A: o caso do município de Osório/RS. 2011. 182p. Dissertação (Mestrado em Geografia). Instituto de Geociências, Universidade Federal do Rio Grande do Sul.

SOMA. Estudo de impacto ambiental: tratamento e disposição de resíduos não-perigosos de Uberaba/MG. 2012.

STEINEMANN, A. Improving alternatives for environmental impact assessment. Environmental Impact Assessment Review, v.21, p.3-21, 2001.

VIASOLO. Estudo de impacto ambiental: aterro sanitário e unidade de compostagem de Betim/MG.2009.

VIASOLO. Estudo de impacto ambiental: central de tratamento de resíduos sólidos Montes Claros/MG, 2011.

ZUBAIR, L. Challenges for environmental impact assessment in Sri Lanka. Environmental Impact Assessment Review, v.21, n.5, p.469-478, 2001. 